\title{
Transmission problem for hyperbolic thermoelastic systems
}

\author{
Luci Harue Fatori, Edson Lueders \\ Department of Mathematics, Universidade Estadual de Londrina, \\ 86.051-990 Londrina-PR,Brazil. \\ Jaime E. Muñoz Rivera \\ National Laboratory for Scientific Computation, \\ Rua Getulio Vargas 333, Quitandinha 25651-070, \\ Petrópolis RJ, Brazil, \\ IM, Federal University of Rio de Janeiro.
}

\begin{abstract}
In this paper we study a transmission problem in thermoelasticity. We show that the linear system is well posed and that the solution decays exponentially to zero as time goes to infinity. That is, denoting by $E(t)$ the first order energy associated to the thermoelastic system, then there exists positive constants $c$ and $\gamma$ such that

$$
E(t) \leq c E(0) e^{-\gamma t}
$$
\end{abstract}

\section{Introduction}

In this paper we consider the asymptotic behaviour of one dimensional bodies which are composed of two different types of materials, one of them is of thermoelastic type, while the other has no thermal effect. That is, we have a material with a localized thermal effect. Since the body is composed of two different types of material the density is not necessarily a continuous function and since the stress strain relation changes from the thermoelastic part to the elastic part, the corresponding model is not a continuous one. The mathematical model which deals with the above situation is called a transmission problem.

Concerning the thermal effect, in the classical linear theory of thermoelasticity the Fourier's law is used to describe the heat conduction in the body. This theory has two principal shortcomings. First, it is unable to account for memory effect which may prevail in some materials, particularly at low temperatures. Secondly, the correspondig parabolic part of the system predicts an unrealistic result, that a thermal disturbance at one point of the body is instantly felt 
every where in the body. These observations lead one to believe that for materials with memory, Fourier's law is not a good model and we have to look for another more general constitutive assumption relating the heat flux to the material thermal history.

We overcome the above shortcomings following the Gurtin-Pipkin's model [4] for the temperature. In this case the thermoelastic system is fully hyperbolic, which in particular implies the finite speed of propagation of the thermal disturbances which is physically more realistic. Therefore we consider the elastic - thermoelastic trasmission problem, for which the thermal part does not depend of the present values of the temperature. Hence the constitutive assumptions we use in this paper are

$$
\begin{aligned}
\sigma(x, t) & =b(x) \varepsilon(x, t)-c(x) \theta(x, t) \\
q(x, t) & =-\int_{0}^{\infty} k(s) \theta_{x}(x, t-s) d s \\
e(x, t) & =c_{u} \theta(x, t)+\alpha u_{x}(x, t)
\end{aligned}
$$

where $\sigma$ is the axial stress, $\varepsilon=u_{x}$ is the deformation, $q$ is the heat flux, $e$ is the internal energy and $\theta$ the temperature difference from the reference value. The memory kernel $k$ is assumed to be a regular functions decaying to zero as time goes to infinity. The functions $b$ and $c$ are discontinuous function of type

$$
b(x)=\left\{\begin{array}{l}
\frac{a}{\rho_{1}} \text { for } \quad 0 \leq x \leq L_{0} \\
\frac{\beta}{\rho_{2}} \text { for } \quad L_{0} \leq x \leq L .
\end{array} \quad c(x)=\left\{\begin{array}{lll}
\frac{\alpha}{\rho_{1}} & \text { for } & 0 \leq x \leq L_{0} \\
0 & \text { for } & L_{0} \leq x \leq L .
\end{array}\right.\right.
$$

Let us denote by

$$
\mathcal{U}(x, t)=\left\{\begin{array}{ll}
u(x, t) & \text { for } \quad 0 \leq x \leq L_{0} \\
v(x, t) & \text { for } \quad L_{0} \leq x \leq L
\end{array} \quad p(x)= \begin{cases}\rho_{1} & \text { for } \quad 0 \leq x \leq L_{0} \\
\rho_{2} & \text { for } \quad L_{0} \leq x \leq L\end{cases}\right.
$$

we see that the couple $(\mathcal{U}, \theta)$ satisfies

$$
\begin{array}{rlll}
\mathcal{U}_{t t}-\left(b(x) \mathcal{U}_{x}\right)_{x}+c(x) \theta_{x} & =0 & \text { a.e. in } & ] 0, L[\times] 0, T[ \\
\theta_{t}-\kappa * \theta_{x x}-\alpha \mathcal{U}_{x t} & =0 & \text { a.e. in } & ] 0, L_{0}[\times] 0, T[
\end{array}
$$

which is the thermoelastic system with discontinuous coefficient. By $*$ we are denoting the convolution product, that is $k * g(\cdot, t)=\int_{0}^{t} k(t-\tau) g(\cdot, \tau) d \tau$. The transmission condition is given by

$$
\left[p(x)\left(b(x) \mathcal{U}_{x}-c(x) \theta\right)\right]=0
$$

where by $[v]$ we are denoting the jump in the function $v$ on $L_{0}$. Note that there exists another implicit transmission condition given by the continuity of the function $\mathcal{U}$, this means that $u\left(L_{0}, t\right)=v\left(L_{0}, t\right)$ for any $t \geq 0$. 
Note that the dissipation given by the thermal effect is only effective over the interval $\left[0, L_{0}\right]$. Other important point in the above model is that the dissipation does not depend on present values of the temperature. These two above properties makes that the corresponding dissipative effect is weaker than the dissipation given by the thermal effect in classic thermoelasticity when the heat flux follows the Fourier law and also more interesting from the mathematical point of view. To facilitate our calculations we rewrite the above system in terms on $u$ and $v$ and without lost of generality we considered $\rho_{1}=\rho_{2}=1$.

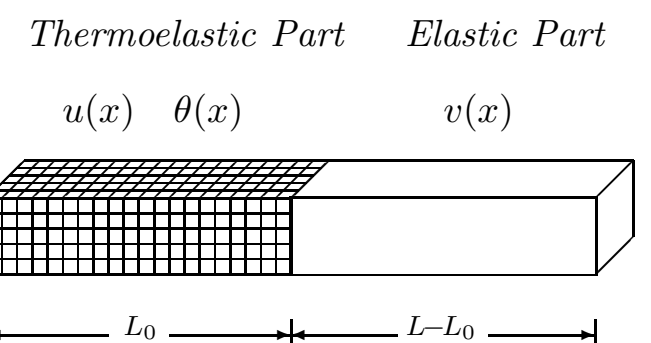

Therefore we have

$$
\begin{array}{rcccc}
u_{t t}-a u_{x x}+\alpha \theta_{x}= & 0 & \text { in } & ] 0, L_{0}\left[\times \mathbb{R}^{+}\right. \\
\theta_{t}-k * \theta_{x x}+\alpha u_{x t}= & 0 & \text { in } & ] 0, L_{0}\left[\times \mathbb{R}^{+}\right. \\
v_{t t}-\beta v_{x x}= & 0 & \text { in } & ] L_{0}, L\left[\times \mathbb{R}^{+}\right. \\
& & & \\
u(x, 0)=u_{0}(x), & u_{t}(x, 0)=u_{1}(x), \\
\theta(x, 0)=\theta_{0}(x), & \\
v(x, 0)=v_{0}(x), & v_{t}(x, 0)=v_{1}(x),
\end{array}
$$

with the following boundary condition

$$
u(0, t)=\theta(0, t)=v(L, t)=0, \quad \theta_{x}\left(L_{0}, t\right)=0 \quad \forall t>0 .
$$

and transmission condition

$$
\begin{aligned}
a u_{x}\left(L_{0}, t\right)-\alpha \theta\left(L_{0}, t\right) & =\beta v_{x}\left(L_{0}, t\right) \quad \forall t>0 \\
u\left(L_{0}, t\right) & =v\left(L_{0}, t\right) \quad \forall t>0
\end{aligned}
$$

For simplicity we consider that the initial past history is fixed and equals to zero,

$$
\theta(x, t)=0, \quad \forall t<0^{-}
$$

The main result of this paper is to show that the localized dissipation due to the thermal effect is strong enough to produce exponential decay to zero of the energy. That is we will prove that 
the solution decays exponentially to zero as time goes to infinity, provided the kernel is positive definite (see [10]) and decays exponentially to zero. The method we use is based on the energy method, combined with compactness arguments. The fundamental tool to get the exponential stability is to construct a functional $\mathcal{F}$, equivalent to the first order energy times $e^{2 \gamma t}$, bounded for $\gamma$ small enought (this implies the exponential decay of the energy). The main difficulty we had is to deal with boundary terms and also to get pointwise estimates at the transmission point $L_{0}$. We overcome such problems using some observabilities estimates see Lions [8].

The rest of this paper is organized as follows. In the next section 2 we show the existence and regularity of the solution. Finally, in section 3 we show the main result of this paper: The exponential decay of the solution.

\section{Existence and Regularity}

In this section we prove the existence and regularity of weak solutions of the hyperbolic thermoelastic equation with memory. We assume that $k$ is a strongly positive definite kernel satisfying

$$
k^{\prime}(t)<0, \quad k^{\prime \prime}(t)>0 \quad \forall t>0 .
$$

Our starting point is to establish the following Lemma whose prove can be found in [10]

Lemma 0.1 Let us suppose that $k \in L^{1}\left(\mathbb{R}^{+}\right)$is a strongly positive definite kernel satisfying $k^{\prime} \in L^{1}\left(\mathbb{R}^{+}\right)$; then we have

$$
\int_{0}^{t}|k * y(\tau)|^{2} d \tau \leq \beta_{0} K \int_{0}^{t} k * y(\tau) y(\tau) d \tau
$$

for any $y \in L_{\text {loc }}^{1}\left(\mathbb{R}^{+}\right)$, where $K=|k|_{1}^{2}+4\left|k^{\prime}\right|_{1}^{2}$ and $\beta_{0}>0$ is such that the function $k(t)-\beta_{0}^{-1} e^{-t}$ is a positive definite kernel.

Let us introduce the following space

$$
\begin{aligned}
\mathcal{V} & =\left\{(\varphi, \psi) \in H^{1}\left(0, L_{0}\right) \times H^{1}\left(L_{0}, L\right) ; \varphi(0)=\psi(L)=0 \text { and } \varphi\left(L_{0}\right)=\psi\left(L_{0}\right)\right\} \\
\mathcal{W} & =\left\{w \in H^{1}\left(0, L_{0}\right) ; w(0)=0\right\}
\end{aligned}
$$

To simplify our analysis let us define the binary operator

$$
k \square g(t)=\int_{0}^{t} k(t-\tau) \int_{0}^{L}|g(x, t)-g(x, \tau)|^{2} d x d \tau .
$$

Under this notation we have 
Lemma 0.2 For any $g \in C\left([0, T] ; H^{1}(0, L)\right)$ and $\eta \in C^{1}(\mathbb{R})$

$$
\begin{aligned}
\int_{0}^{L} \int_{0}^{t} \eta(t-\tau) g(x, \tau) d \tau g_{t}(x, t) d x & =-\frac{1}{2} \eta(t) \int_{0}^{L}|g|^{2} d x+\frac{1}{2} \eta^{\prime} \square g- \\
& -\frac{1}{2} \frac{d}{d t}\left\{\eta \square g-\left(\int_{0}^{t} \eta d \tau\right) \int_{0}^{L}|g|^{2} d x\right\} .
\end{aligned}
$$

Proof: To show the above identity, it is enough to differentiate with respect to the time the expression $\eta \square g$.

The definition of weak solution we use in this work is given as follows

Definition 0.1 We say that $(u, v, \theta)$ is a weak solution of the system (1)-(3) when

$$
\begin{aligned}
(u, v) & \in L^{\infty}(0, T ; \mathcal{V}) \cap W^{1, \infty}\left(0, T ; L^{2}\left(0, L_{0}\right) \times L^{2}\left(L_{0}, L\right)\right) \\
\theta & \in L^{\infty}\left(0, T ; L^{2}\left(0, L_{0}\right)\right) \quad k * \theta_{x} \in L^{2}\left(0, T ; L^{2}\left(0, L_{0}\right)\right)
\end{aligned}
$$

and satisfies the following identities

$$
\begin{gathered}
\int_{0}^{T} \int_{0}^{L_{0}}\left\{u \Phi_{t t}+a u_{x} \Phi_{x}-\alpha \theta \Phi_{x}\right\} d x d t+\int_{0}^{T} \int_{L_{0}}^{L}\left\{v \Psi_{t t}+\beta v_{x} \Psi_{x}\right\} d x d t= \\
\int_{0}^{L_{0}} u_{1} \Phi(0)-u_{0} \Phi_{t}(0) d x+\int_{L_{0}}^{L} v_{1} \Psi(0)-v_{0} \Psi_{t}(0) d x . \\
-\int_{0}^{T} \int_{0}^{L_{0}}\left\{\theta \Gamma_{t}-k * \theta_{x} \Gamma_{x}+\alpha u_{x} \Gamma_{t}\right\} d x d t=\int_{0}^{L_{0}} \theta_{0} \Gamma(0) d x+\alpha \int_{0}^{L_{0}} u_{x}(0) \Gamma(0) d x
\end{gathered}
$$

for any $(\Phi, \Psi) \in C^{2}([0, T] ; \mathcal{V})$ and $\Gamma \in C^{1}([0, T] ; \mathcal{W})$ such that $\Phi(T)=\Phi_{t}(T)=\Psi(T)=\Psi_{t}(T)=$ $\Gamma(T)=0$.

Let us introduce the energy functions

$$
\begin{aligned}
& E_{1}(t ; u, \theta):=\frac{1}{2} \int_{0}^{L_{0}}\left|u_{t}\right|^{2}+a\left|u_{x}\right|^{2}+|\theta|^{2} d x \\
& E_{2}(t ; v):=\frac{1}{2} \int_{L_{0}}^{L}\left|v_{t}\right|^{2}+\beta\left|v_{x}\right|^{2} d x \\
& E(t ; u, \theta, v):=E_{1}(t ; u, \theta)+E_{2}(t ; v) \\
& F(t ; u, \theta, v):=\frac{1}{2} \int_{0}^{L_{0}}\left|u_{t t}\right|^{2}+a\left|u_{x t}\right|^{2}+\left|\theta_{t}\right|^{2}+k(t)\left|\theta_{x}\right|^{2}-k^{\prime} \square \theta_{x} d x \\
&+\frac{1}{2} \int_{L_{0}}^{L}\left|v_{t t}\right|^{2}+\beta\left|v_{x t}\right|^{2} d x .
\end{aligned}
$$

In these conditions we are able to prove the following Theorem 
Theorem 0.1 Let us suppose that the initial data satisfies $\left(u_{0}, v_{0}\right) \in \mathcal{V}, \quad\left(u_{1}, v_{1}\right) \in L^{2}\left(0, L_{0}\right) \times$ $L^{2}\left(L_{0}, L\right)$ and $\theta_{0} \in L^{2}\left(0, L_{0}\right)$. Then there exists a unique weak solution for (1)-(3). Moreover if we take the initial data $\left(u_{0}, v_{0}\right) \in\left[H^{2}\left(0, L_{0}\right) \times H^{2}\left(L_{0}, L\right)\right] \cap \mathcal{V},\left(u_{1}, v_{1}\right) \in \mathcal{V}, \theta_{0} \in H^{1}\left(0, L_{0}\right)$ verifying the compatibility condition given by

$$
a u_{0, x}\left(L_{0}\right)-\alpha \theta_{0}\left(L_{0}\right)=\beta v_{0, x}\left(L_{0}\right)
$$

then the solution satisfies,

$$
\begin{aligned}
(u, v) & \in \bigcap_{k=0}^{2} W^{k, \infty}\left(0, T ; H^{2-k}\left(0, L_{0}\right) \times H^{2-k}\left(L_{0}, L\right)\right) \\
\theta & \in \bigcap_{k=0}^{1} W^{k, \infty}\left(0, T ; H^{1-k}\left(0, L_{0}\right)\right) \\
k * \theta_{x x} & \in L^{\infty}\left(0, T ; L^{2}\left(0, L_{0}\right)\right)
\end{aligned}
$$

Proof: Let us denote $\left\{\left(\varphi_{j}, \psi_{j}\right)\right\} \times\left\{w_{j}\right\}$ an orthonormal basis of $\mathcal{V} \times \mathcal{W}$ and

$$
\left(u^{m}(t), v^{m}(t)\right)=\sum_{j=1}^{m} h_{j, m}(t)\left(\varphi_{j}, \psi_{j}\right), \quad \theta^{m}(t)=\sum_{j=1}^{m} g_{j, m}(t) w_{j}
$$

where the functions $h_{j, m}(t)$ and $g_{j, m}(t)$ are given by the solution of the approximated system,

$$
\begin{array}{r}
\int_{0}^{L_{0}}\left[u_{t t}^{m} \varphi_{j}+a u_{x}^{m} \varphi_{j, x}-\alpha \theta^{m} \varphi_{j, x}\right] d x+\int_{L_{0}}^{L}\left[v_{t t}^{m} \psi_{j}+\beta v_{x}^{m} \psi_{j, x}\right] d x=0 \\
\int_{0}^{L_{0}}\left[\theta_{t}^{m} w_{j}+k * \theta_{x}^{m} w_{j, x}-\alpha u_{t}^{m} w_{j, x}\right] d x=0
\end{array}
$$

with initial data

$$
\left(u^{m}(0), v^{m}(0)\right)=\left(u_{0}^{m}, v_{0}^{m}\right), \quad\left(u_{t}^{m}(0), v_{t}^{m}(0)\right)=\left(u_{1}^{m}, v_{1}^{m}\right), \quad \theta^{m}(0)=\theta_{0}^{m} .
$$

Where

$$
\left(u_{0}^{m}, v_{0}^{m}\right)=\sum_{i=1}^{m} \alpha_{i}\left(\varphi_{i}, \psi_{i}\right), \quad\left(u_{1}^{m}, v_{1}^{m}\right)=\sum_{i=1}^{m} \beta_{i}\left(\varphi_{i}, \psi_{i}\right), \quad \theta_{0}^{m}=\sum_{i=1}^{m} \gamma_{i} w_{i}
$$

are such that

$$
\left(u_{0}^{m}, v_{0}^{m}\right) \quad \rightarrow \quad\left(u_{0}, v_{0}\right), \quad\left(u_{1}^{m}, v_{1}^{m}\right) \quad \rightarrow \quad\left(u_{1}, v_{1}\right), \quad \theta_{0}^{m} \quad \rightarrow \quad \theta_{0}
$$

strongly in their corresponding spaces. The existence of approximated solutions $u^{m}, \theta^{m}$ and $v^{m}$ are guaranteed by standard results on Ordinary Differential Equations. Our next step is to show that the approximated solution remains bounded for any $m>0$. To this end, let us multiply equation (5) by $h_{j, m}^{\prime}(t)$ and (6) by $g_{j, m}(t)$, summing up the product result in $j$ we arrive at

$$
\frac{d}{d t} E\left(t ; u^{m}, \theta^{m}, v^{m}\right)=-\int_{0}^{L_{0}} k * \theta_{x}^{m} \theta_{x}^{m} d x
$$


Integrating from 0 to $t$ the above relation and using Lemma 0.1 it follows that there exists a positive constant $C_{0}$ satisfying

$$
E\left(t ; u^{m}, \theta^{m}, v^{m}\right)+C_{0} \int_{0}^{t} \int_{0}^{L_{0}}\left|k * \theta_{x}^{m}\right|^{2} d x d \tau \leq E\left(0 ; u^{m}, \theta^{m}, v^{m}\right) .
$$

From our choice of initial data it follows that

$$
\begin{aligned}
E\left(t ; u^{m}, \theta^{m}, v^{m}\right) & \text { is bounded for any } m \in \mathbb{N} \\
k * \theta_{x}^{m} & \text { is bounded in } L^{2}\left(0, T ; L^{2}\left(0, L_{0}\right)\right) .
\end{aligned}
$$

Differentiating equation (5) and (6) with respect to the time we get

$$
\begin{gathered}
\int_{0}^{L_{0}}\left[u_{t t t}^{m} \varphi_{j}+a u_{x t}^{m} \varphi_{j, x}-\alpha \theta_{t}^{m} \varphi_{j, x}\right] d x+\int_{L_{0}}^{L}\left[v_{t t t}^{m} \psi_{j}+\beta v_{x t}^{m} \psi_{j, x}\right] d x=0 \\
\int_{0}^{L_{0}}\left[\theta_{t t}^{m} w_{j}+k(0) \theta_{x}^{m} w_{j, x}+k^{\prime} * \theta_{x}^{m} w_{j, x}-\alpha u_{t t}^{m} w_{j, x}\right] d x=0 .
\end{gathered}
$$

Multiplying equation (10) by $h_{j, m}^{\prime \prime}$ and (11) by $g_{j, m}^{\prime}$ we obtain

$$
\begin{aligned}
\frac{d}{d t} \frac{1}{2}\left[\int_{0}^{L_{0}}\left|u_{t t}^{m}\right|^{2}+a\left|u_{x t}^{m}\right|^{2}+\left|\theta_{t}^{m}\right|^{2}\right. & \left.+k(0)\left|\theta_{x}^{m}\right|^{2} d x+\int_{L_{0}}^{L}\left|v_{t t}^{m}\right|^{2}+\beta\left|v_{x t}^{m}\right|^{2} d x\right]= \\
& =-\int_{0}^{L_{0}} \int_{0}^{t} k^{\prime}(t-\tau) \theta_{x}^{m}(x, \tau) d \tau \theta_{x t}^{m}(x, t) d x .
\end{aligned}
$$

Using Lemma 0.2 and hypotheses (4) we conclude that

$$
\frac{d}{d t} F\left(t ; u^{m}, \theta^{m}, v^{m}\right)=\frac{k^{\prime}(t)}{2} \int_{0}^{L_{0}}\left|\theta_{x}^{m}\right|^{2} d x-\frac{1}{2} k^{\prime \prime} \square \theta_{x}^{m}<0 .
$$

Integrating from 0 to $t$ the above relation we get

$$
\begin{aligned}
F\left(t ; u^{m}, \theta^{m}, v^{m}\right) & \leq \frac{1}{2} \int_{0}^{L_{0}}\left|u_{t t}^{m}(0)\right|^{2}+a\left|u_{1, x}^{m}\right|^{2}+\left|\theta_{t}^{m}(0)\right|^{2}+k(0)\left|\theta_{0, x}^{m}\right|^{2} d x \\
& +\frac{1}{2} \int_{L_{0}}^{L}\left|v_{t t}^{m}(0)\right|^{2}+\beta\left|v_{1, x}^{m}\right|^{2} d x
\end{aligned}
$$

Now, we do $t \rightarrow 0^{+}$in (5) and (6) and multiplying the limit result by $h_{j, m}^{\prime \prime}(0)$ and $g_{j, m}^{\prime}(0)$ respectively. Summing up in $j$ and using compatibility condition we get

$$
\begin{aligned}
\int_{0}^{L_{0}}\left|u_{t t}^{m}(0)\right|^{2}+\left|\theta_{t}^{m}(0)\right|^{2} d x+\int_{L_{0}}^{L}\left|v_{t t}^{m}(0)\right|^{2} d x & =\int_{L_{0}}^{L} \beta v_{0, x x}^{m} v_{t t}^{m}(0) d x \\
& +\int_{0}^{L_{0}}\left\{a u_{0, x x}^{m}-\alpha \theta_{0, x}^{m}\right\} u_{t t}^{m}(0)-\alpha u_{1, x}^{m} \theta_{t}^{m}(0) d x .
\end{aligned}
$$

Applying Young's inequality we have

$$
\left(u_{t t}^{m}(0), v_{t t}^{m}(0), \theta_{t}^{m}(0)\right) \quad \text { is bounded in } \quad L^{2}\left(0, L_{0}\right) \times L^{2}\left(L_{0}, L\right) \times L^{2}\left(0, L_{0}\right) .
$$


Therefore

$$
F\left(t ; u^{m}, \theta^{m}, v^{m}\right) \text { is bounded for any } m \in \mathbb{N} \text {. }
$$

From (8) and (12) we get that $(u, \theta, v)$ obtained taking the limit when $m \rightarrow \infty$ is such that

$$
\begin{aligned}
(u, v), \quad\left(u_{t}, v_{t}\right) & \in L^{\infty}(0, T ; \mathcal{V}) \\
\left(u_{t t}, v_{t t}\right) & \in L^{\infty}\left(L^{2}\left(0, L_{0}\right) \times L^{2}\left(L_{0}, L\right)\right) \\
\left(\theta, \theta_{t}\right) & \in L^{\infty}\left(H^{1}\left(0, L_{0}\right) \times L^{2}\left(0, L_{0}\right)\right) \\
k * \theta_{x} & \in L^{2}\left(0, T ; L^{2}\left(0, L_{0}\right)\right)
\end{aligned}
$$

and satisfies

$$
\begin{aligned}
a u_{x x} & =u_{t t}+\alpha \theta_{x} \\
k * \theta_{x x} & =\theta_{t}+\alpha u_{x t} \\
\beta v_{x x} & =v_{t t}
\end{aligned}
$$

from this we conclude that

$$
\begin{aligned}
\left(u_{x x}, v_{x x}\right) & \in L^{\infty}\left(0, T ; L^{2}\left(0, L_{0}\right) \times L^{2}\left(L_{0}, L\right)\right) \\
k * \theta_{x x} & \in L^{\infty}\left(0, T ; L^{2}\left(0, L_{0}\right) .\right.
\end{aligned}
$$

The rest of proof is a matter of routine.

\section{Uniform rate of decay}

In this section we show that the solution of the system (1)-(2) decays exponentially as time goes to infinity. The method we use here is based on the construction of a functional $\mathcal{L}(t)$ equivalent to the first order energy whose derivative is negative proportional to itself. Here we will assume that $k$ and $-k^{\prime}$ are positive definite kernel satisfying

$$
\begin{gathered}
k^{\prime}, k^{\prime \prime} \in C^{1}\left(\mathbb{R}^{+}\right) \\
\int_{0}^{T} k^{\prime} * \phi \phi d t \leq-C \int_{0}^{T} k * \phi \phi d t .
\end{gathered}
$$

The above inequality is satisfied for any function $k$ which is sum of exponential functions with varying rates of decay. Since the kernel $k$ decays exponentially, we take $\gamma>0$ small enough such that there exists $\varepsilon>0$ satisfying

$$
K(t):=e^{\gamma t} k(t) \leq C e^{-\varepsilon t}
$$


Let us denote $U(x, t)=u(x, t) e^{\gamma t}, \Theta(x, t)=\theta(x, t) e^{\gamma t}$ and $V(x, t)=v(x, t) e^{\gamma t}$, then $(U, \Theta, V)$ satisfies:

$$
\begin{aligned}
& U_{t t}-a U_{x x}+\alpha \Theta_{x}=R \\
& \Theta_{t}-K * \Theta_{x x}+\alpha U_{x t}=S, \\
& V_{t t}-\beta V_{x x}=Q
\end{aligned}
$$

where $R, S$ and $Q$ are given by:

$$
R:=2 \gamma U_{t}-\gamma^{2} U, \quad S:=\gamma \Theta+\alpha \gamma U_{x}, \quad Q:=2 \gamma V_{t}-\gamma^{2} V .
$$

with $U, \Theta$ and $V$ satisfying the same boundary conditions and initial values as in system (1)-(3). Let us introduce the energy function

$$
\begin{aligned}
E_{1}(t ; U, \Theta) & :=\frac{1}{2} \int_{0}^{L_{0}}\left|U_{t}\right|^{2}+a\left|U_{x}\right|^{2}+|\Theta|^{2} d x \\
E_{2}(t ; V) & :=\frac{1}{2} \int_{L_{0}}^{L}\left|V_{t}\right|^{2}+\beta\left|V_{x}\right|^{2} d x \\
E(t ; U, \Theta, V) & :=E_{1}(t ; U, \Theta)+E_{2}(t ; V) .
\end{aligned}
$$

To show the exponential decay of the solution $(u, \theta, v)$ it is enough to prove that $E(t, U, \Theta, V)$ is bounded. To this end, we start with the following Lemma.

Lemma 0.3 Let us suppose that the initial data satisfy

$$
\left(u_{0}, v_{0}\right) \in\left[H^{2}\left(0, L_{0}\right) \times H^{2}\left(L_{0}, L\right)\right] \cap \mathcal{V}, \quad\left(u_{1}, v_{1}\right) \in \mathcal{V}, \quad \theta_{0} \in H^{1}\left(0, L_{0}\right)
$$

verifying the compatibility condition given by

$$
a u_{0, x}\left(L_{0}\right)-\alpha \theta_{0}\left(L_{0}\right)=\beta v_{0, x}\left(L_{0}\right)
$$

then we have

$$
\frac{d}{d t} E(t ; U, \Theta, V) \leq-\int_{0}^{L_{0}} K * \Theta_{x} \Theta_{x} d x+c \gamma E(t ; U, \Theta, V)
$$

Proof: Multiplying equation (15) by $U_{t}$ and equation (16) by $\Theta$ and integrating from 0 to $L_{0}$ we get

$$
\begin{aligned}
\frac{d}{d t} E_{1}(t ; U, \Theta)= & -\int_{0}^{L_{0}} K * \Theta_{x} \Theta_{x} d x+\int_{0}^{L_{0}}\left(R U_{t}+S \Theta\right) d x \\
& +a U_{x}\left(L_{0}, t\right) U_{t}\left(L_{0}, t\right)-\alpha \Theta\left(L_{0}, t\right) V_{t}\left(L_{0}, t\right) .
\end{aligned}
$$

On the other hand, multiplying equation (17) by $V_{t}$ and integrating from $L_{0}$ to $L$ we get

$$
\frac{d}{d t} E_{2}(t ; V):=\int_{L_{0}}^{L} V_{t} Q d x-\beta V_{x}\left(L_{0}, t\right) V_{t}\left(L_{0}, t\right)
$$


After applying Holder's inequality and Young's inequality we have

$$
\begin{aligned}
\left|\int_{0}^{L_{0}} R U_{t} d x\right| & \leq c \gamma\left(\int_{0}^{L_{0}}\left|U_{t}\right|^{2} d x+\int_{0}^{L_{0}}\left|U_{x}\right|^{2} d x\right) \\
\left|\int_{0}^{L_{0}} S \Theta d x\right| & \leq c \gamma\left(\int_{0}^{L_{0}}|\Theta|^{2} d x+\int_{0}^{L_{0}}\left|U_{x}\right|^{2} d x\right) \\
\left|\int_{L_{0}}^{L} V_{t} Q d x\right| & \leq c \gamma\left(\int_{L_{0}}^{L}\left|V_{t}\right|^{2} d x+\int_{L_{0}}^{L}\left|V_{x}\right|^{2} d x\right)
\end{aligned}
$$

From now on $c$ will denote a positive constant, easy to estimate, which can be different in different places. Thereby, from transmission's conditions our conclusion follows

Lemma 0.4 With the same hypotheses as Lemma 0.3 we have

$$
\begin{aligned}
-\frac{d}{d t} \int_{0}^{L_{0}} K * \Theta \Theta d x= & -K(0) \int_{0}^{L_{0}}|\Theta|^{2} d x+\int_{0}^{L_{0}}\left|K * \Theta_{x}\right|^{2} d x \\
& +\alpha U_{t}\left(L_{0}, t\right) K * \Theta\left(L_{0}, t\right)-\int_{0}^{L_{0}} \Theta K^{\prime} * \Theta d x \\
& \quad-\alpha \int_{0}^{L_{0}} U_{t} K * \Theta_{x} d x-\int_{0}^{L_{0}} S(K * \Theta) d x \\
\frac{d}{d t} \int_{0}^{L_{0}} U U_{t} d x \leq- & \frac{a}{2} \int_{0}^{L_{0}}\left|U_{x}\right|^{2} d x+\int_{0}^{L_{0}}\left|U_{t}\right|^{2} d x \\
+ & \frac{\alpha^{2}}{2 a} \int_{0}^{L_{0}}|\Theta|^{2} d x+\left\{\frac{24 a}{L_{0}}+\frac{24 \alpha^{2}}{L_{0}}\right\}\left|U\left(L_{0}, t\right)\right|^{2} \\
+ & \frac{L_{0}}{96}\left\{a\left|U_{x}\left(L_{0}, t\right)\right|^{2}+\left|\Theta\left(L_{0}, t\right)\right|^{2}\right\}+c \gamma E_{1}(t ; U, \Theta) . \\
\frac{d}{d t} \int_{L_{0}}^{L}(x-L) V_{x} V_{t} d x \leq & \frac{\lambda_{0}}{2}\left\{a\left|U_{x}\left(L_{0}, t\right)\right|^{2}+\left|U_{t}\left(L_{0}, t\right)\right|^{2}+\left|\Theta\left(L_{0}, t\right)\right|^{2}\right\} \\
& -E_{2}(t ; V)+c \gamma E_{2}(t ; V)
\end{aligned}
$$

where $\lambda_{0}=\left(L-L_{0}\right) \max \left\{1, \frac{\bar{c}}{\beta}\right\}$ and $\bar{c}=\max \left\{\frac{a}{2}, \frac{\alpha^{2}}{2}\right\}$.

Proof: We have

$$
\begin{aligned}
\frac{d}{d t} \int_{0}^{L_{0}} \Theta(K * \Theta) d x= & \int_{0}^{L_{0}} \Theta_{t} K * \Theta d x+K(0) \int_{0}^{L_{0}}|\Theta|^{2} d x+\int_{0}^{L_{0}} K^{\prime} * \Theta \Theta d x \\
= & K(0) \int_{0}^{L_{0}}|\Theta|^{2} d x+\int_{0}^{L_{0}} K * \Theta_{x x}(K * \Theta) d x-\alpha \int_{0}^{L_{0}} U_{x t}(K * \Theta) d x \\
& +\int_{0}^{L_{0}} S(K * \Theta) d x+\int_{0}^{L_{0}} K^{\prime} * \Theta \Theta d x \\
= & K(0) \int_{0}^{L_{0}}|\Theta|^{2} d x-\int_{0}^{L_{0}}\left|K * \Theta_{x}\right|^{2} d x-\alpha U_{t}\left(L_{0}, t\right) K * \Theta\left(L_{0}, t\right) \\
& +\int_{0}^{L_{0}} S(K * \Theta) d x+\int_{0}^{L_{0}} K^{\prime} * \Theta \Theta d x+\alpha \int_{0}^{L_{0}} U_{t} K * \Theta_{x} d x .
\end{aligned}
$$


On the other hand from (15) we have

$$
\begin{aligned}
\frac{d}{d t} \int_{0}^{L_{0}} U U_{t} d x= & \int_{0}^{L_{0}}\left|U_{t}\right|^{2} d x+\int_{0}^{L_{0}} U U_{t t} d x \\
= & \int_{0}^{L_{0}}\left|U_{t}\right|^{2} d x+a \int_{0}^{L_{0}} U U_{x x} d x-\alpha \int_{0}^{L_{0}} U \Theta_{x} d x+\int_{0}^{L_{0}} R U d x \\
= & \int_{0}^{L_{0}}\left|U_{t}\right|^{2} d x+a U\left(L_{0}, t\right) U_{x}\left(L_{0}, t\right)-\alpha U\left(L_{0}, t\right) \Theta\left(L_{0}, t\right) \\
& -a \int_{0}^{L_{0}}\left|U_{x}\right|^{2} d x+\alpha \int_{0}^{L_{0}} U_{x} \Theta d x+\int_{0}^{L_{0}} R U d x \\
\leq & -\frac{a}{2} \int_{0}^{L_{0}}\left|U_{x}\right|^{2} d x+\int_{0}^{L_{0}}\left|U_{t}\right|^{2} d x+\frac{\alpha^{2}}{2 a} \int_{0}^{L_{0}}|\Theta|^{2} d x \\
& +a U\left(L_{0}, t\right) U_{x}\left(L_{0}, t\right)-\alpha U\left(L_{0}, t\right) \Theta\left(L_{0}, t\right)+\int_{0}^{L_{0}} R U d x .
\end{aligned}
$$

Since

$$
\begin{aligned}
& \left|a U\left(L_{0}, t\right) U_{x}\left(L_{0}, t\right)\right| \leq \frac{24 a}{L_{0}}\left|U\left(L_{0}, t\right)\right|^{2}+\frac{a L_{0}}{96}\left|U_{x}\left(L_{0}, t\right)\right|^{2} \\
& \left|\alpha U\left(L_{0}, t\right) \Theta\left(L_{0}, t\right)\right| \leq \frac{24 \alpha^{2}}{L_{0}}\left|U\left(L_{0}, t\right)\right|^{2}+\frac{L_{0}}{96}\left|\Theta\left(L_{0}, t\right)\right|^{2} .
\end{aligned}
$$

Therefore

$$
\begin{aligned}
\frac{d}{d t} \int_{0}^{L_{0}} U U_{t} d x \leq & -\frac{a}{2} \int_{0}^{L_{0}}\left|U_{x}\right|^{2} d x+\int_{0}^{L_{0}}\left|U_{t}\right|^{2} d x+ \\
& +\frac{\alpha^{2}}{2 a} \int_{0}^{L_{0}}|\Theta|^{2} d x+\left\{\frac{24 a}{L_{0}}+\frac{24 \alpha^{2}}{L_{0}}\right\}\left|U\left(L_{0}, t\right)\right|^{2} \\
& +\frac{L_{0}}{96}\left\{a\left|U_{x}\left(L_{0}, t\right)\right|^{2}+\left|\Theta\left(L_{0}, t\right)\right|^{2}\right\}+c \gamma E_{1}(t ; U, \Theta) .
\end{aligned}
$$

Now we proof the last estimate,

$$
\begin{aligned}
\frac{d}{d t} \int_{L_{0}}^{L} \varphi(x) V_{t} V_{x} d x= & \frac{1}{2} \int_{L_{0}}^{L} \varphi(x) \frac{d}{d x}\left|V_{t}\right|^{2} d x+\frac{\beta}{2} \int_{L_{0}}^{L} \varphi(x) \frac{d}{d x}\left|V_{x}\right|^{2} d x \\
& +\int_{L_{0}}^{L} Q \varphi(x) V_{x} d x \\
= & \frac{\varphi\left(L_{0}\right)}{2}\left\{\beta\left|V_{x}\left(L_{0}, t\right)\right|^{2}+\left|V_{t}\left(L_{0}, t\right)\right|^{2}\right\} \\
& -\frac{1}{2} \int_{L_{0}}^{L} \varphi^{\prime}(x)\left\{\left|V_{t}\right|^{2}+\beta\left|V_{x}\right|^{2}\right\} d x+\int_{L_{0}}^{L} Q \varphi(x) V_{x} d x .
\end{aligned}
$$

Taking $\varphi(x)=(x-L)$ we arrive to our conclusion.

Let us introduce the multipliers $q$ and $w$ satisfying

$$
\begin{aligned}
-q_{x x} & =U_{t} & & \text { in }\left(0, L_{0}\right) \times \mathbb{R}^{+} \\
q(0, t) & =q\left(L_{0}, t\right)=0 & & \text { in } \mathbb{R}^{+}
\end{aligned}
$$




$$
\begin{aligned}
-w_{x x} & =\Theta-\bar{\Theta} & & \text { in }\left(0, L_{0}\right) \times \mathbb{R}^{+} \\
w_{x}(0, t) & =w_{x}\left(L_{0}, t\right)=0 & & \text { in } \mathbb{R}^{+}
\end{aligned}
$$

where $\bar{\Theta}=\frac{1}{L_{0}} \int_{0}^{L_{0}} \Theta d x$. We easily obtain

$$
\int_{0}^{L_{0}}\left|w_{x}\right|^{2} d x \leq c \int_{0}^{L_{0}}|\Theta|^{2} d x
$$

Lemma 0.5 With the same hypotheses as Lemma 0.3 we have

$$
\begin{aligned}
\frac{d}{d t} \int_{0}^{L_{0}} q_{x} \Theta d x \leq & -\frac{\alpha}{2} \int_{0}^{L_{0}}\left|U_{t}\right|^{2} d x+\frac{\alpha a}{32} \int_{0}^{L_{0}}\left|U_{x}\right|^{2} d x+\left[\frac{16 c_{p}^{2} \delta_{1} a}{\alpha L_{0}}+\frac{16 a}{\alpha}+\alpha\right] \int_{0}^{L_{0}}|\Theta|^{2} d x \\
& +\left(\frac{64 \alpha \lambda_{1}^{2}}{L_{0}}+\frac{16 \lambda_{1}^{2} K(0)}{\alpha L_{0}}\right)\left\|q_{x}(., t)\right\|_{H^{3 / 4}}^{2}+\frac{\alpha L_{0}}{64 K(0)}\left|K * \Theta_{x}(0, t)\right|^{2} \\
& +\frac{\alpha L_{0}}{256}\left|U_{t}\left(L_{0}, t\right)\right|^{2}+\frac{1}{2 \alpha} \int_{0}^{L_{0}}\left|K * \Theta_{x}\right|^{2} d x+c \gamma E_{1}(t ; U, \Theta)
\end{aligned}
$$

where $\lambda_{1}$ and $\delta_{1}$ are the embedding constants of $H^{3 / 4}\left(0, L_{0}\right) \hookrightarrow L^{\infty}\left(0, L_{0}\right)$ and $L^{2}\left(0, L_{0}\right) \hookrightarrow L^{1}\left(0, L_{0}\right)$, respectively, and $c_{p}$ is a constant satisfying

$$
\left|f\left(L_{0}\right)\right|^{2} \leq c_{p}^{2} \int_{0}^{L}\left|f_{x}\right|^{2} d x, \quad \int_{0}^{L}|f|^{2} d x \leq c_{p}^{2} \int_{0}^{L}\left|f_{x}\right|^{2} d x, \quad \forall f \in \mathcal{W}
$$

Proof. Note that

$$
\begin{aligned}
\frac{d}{d t} \int_{0}^{L_{0}} q_{x} \Theta d x & =\int_{0}^{L_{0}} q_{x t} \Theta d x+\int_{0}^{L_{0}} q_{x} \Theta_{t} d x \\
& =\underbrace{\int_{0}^{L_{0}} q_{x t} \Theta d x}_{I_{1}}+\underbrace{\int_{0}^{L_{0}} q_{x} K * \Theta_{x x} d x}_{I_{2}}-\underbrace{\alpha \int_{0}^{L_{0}} q_{x} U_{x t} d x}_{I_{3}}+\underbrace{\int_{0}^{L_{0}} q_{x} S d x}_{I_{4}} .
\end{aligned}
$$

Recalling the definitions of $q_{x}$ and $w$ we have

$$
\begin{aligned}
I_{1}= & \int_{0}^{L_{0}} q_{x t}(\Theta-\bar{\Theta}) d x=-\int_{0}^{L_{0}} q_{x t} w_{x x} d x=-\int_{0}^{L_{0}} U_{t t} w_{x} d x \\
= & -a \int_{0}^{L_{0}} U_{x x} w_{x} d x+\alpha \int_{0}^{L_{0}} \Theta_{x} w_{x} d x-\int_{0}^{L_{0}} R w_{x} d x \\
= & a \int_{0}^{L_{0}} U_{x} w_{x x} d x-\alpha \int_{0}^{L_{0}} \Theta w_{x x} d x-\int_{0}^{L_{0}} R w_{x} d x \\
= & -a \int_{0}^{L_{0}} U_{x}(\Theta-\bar{\Theta}) d x+\alpha \int_{0}^{L_{0}}|\Theta|^{2} d x-\frac{\alpha}{L_{0}}\left(\int_{0}^{L_{0}} \Theta d x\right)^{2}-\int_{0}^{L_{0}} R w_{x} d x \\
= & -a \int_{0}^{L_{0}} U_{x} \Theta d x+a \bar{\Theta} U\left(L_{0}, t\right)+\alpha \int_{0}^{L_{0}}|\Theta|^{2} d x-\frac{\alpha}{L_{0}}\left(\int_{0}^{L_{0}} \Theta d x\right)^{2} \\
& -\int_{0}^{L_{0}} R w_{x} d x .
\end{aligned}
$$


Since

$$
\begin{aligned}
\left|a \int_{0}^{L_{0}} U_{x} \Theta d x\right| \leq \frac{\alpha a}{64} \int_{0}^{L_{0}}\left|U_{x}\right|^{2} d x+\frac{16 a}{\alpha} \int_{0}^{L_{0}}|\Theta|^{2} d x & \left|U\left(L_{0}, t\right)\right|^{2} \leq c_{p}^{2} \int_{0}^{L_{0}}\left|U_{x}\right|^{2} d x \\
& \int_{0}^{L_{0}} R w_{x} d x \leq c \gamma E_{1}(t ; U, \Theta) \\
\left|a \bar{\Theta} U\left(L_{0}, t\right)\right| \leq & \frac{16 c_{p}^{2} a}{\alpha}|\bar{\Theta}|^{2}+\frac{\alpha a}{64 c_{p}^{2}}\left|U\left(L_{0}, t\right)\right|^{2} \\
& \leq \frac{16 c_{p}^{2} a}{\alpha}\left|\frac{1}{L_{0}} \int_{0}^{L_{0}} \Theta d x\right|^{2}+\frac{\alpha a}{64 c_{p}^{2}}\left|U\left(L_{0}, t\right)\right|^{2} \\
& \leq \frac{16 c_{p}^{2} \delta_{1} a}{\alpha L_{0}^{2}} \int_{0}^{L_{0}}|\Theta|^{2} d x+\frac{\alpha a}{64} \int_{0}^{L_{0}}\left|U_{x}\right|^{2} d x
\end{aligned}
$$

Using theses estimates in (24) we get

$$
I_{1} \leq \frac{\alpha a}{32} \int_{0}^{L_{0}}\left|U_{x}\right|^{2} d x+\left(\frac{16 a}{\alpha}+\frac{16 c_{p}^{2} \delta_{1} a}{\alpha L_{0}^{2}}+\alpha\right) \int_{0}^{L_{0}}|\Theta|^{2} d x+c \gamma E_{1}(t ; U, \Theta)
$$

Now we consider the term

$$
\begin{aligned}
I_{2} & =\left.q_{x} K * \Theta_{x}\right|_{0} ^{L_{0}}-\int_{0}^{L_{0}} q_{x x} K * \Theta_{x} d x \\
& \leq\left\|q_{x}(., t)\right\|_{L^{\infty}}\left|K * \Theta_{x}(0, t)\right|+\int_{0}^{L_{0}} U_{t} K * \Theta_{x} d x \\
& \leq \lambda_{1}\left\|q_{x}(., t)\right\|_{H^{3 / 4}}\left|K * \Theta_{x}(0, t)\right|+\int_{0}^{L_{0}} U_{t} K * \Theta_{x} d x .
\end{aligned}
$$

Applying Holder's inequality and Young's inequality in the last expression we have $I_{2} \leq \frac{\alpha}{2} \int_{0}^{L_{0}}\left|U_{t}\right|^{2} d x+\frac{16 K(0) \lambda_{1}^{2}}{\alpha L_{0}}\left\|q_{x}(., t)\right\|_{H^{3 / 4}}^{2}+\frac{\alpha L_{0}}{64 K(0)}\left|K * \Theta_{x}(0, t)\right|^{2}+\frac{1}{2 \alpha} \int_{0}^{L_{0}}\left|K * \Theta_{x}\right|^{2} d x$.

Analogously note that $I_{3}$ satisfies

$$
\begin{aligned}
I_{3} & =-\alpha \int_{0}^{L_{0}}\left|U_{t}\right|^{2} d x+\left.\alpha q_{x} U_{t}\right|_{0} ^{L_{0}} \\
& \leq-\alpha \int_{0}^{L_{0}}\left|U_{t}\right|^{2} d x+\frac{\alpha L_{0}}{256}\left|U_{t}\left(L_{0}, t\right)\right|^{2}+\frac{64 \alpha \lambda_{1}^{2}}{L_{0}}\left\|q_{x}(., t)\right\|_{H^{3 / 4}}^{2} .
\end{aligned}
$$

Finally, we have that

$$
I_{4}=\int_{0}^{L_{0}} q_{x}\left(\gamma \Theta+\alpha \gamma U_{x}\right) d x \leq c \gamma E_{1}(t ; U, \Theta) .
$$

Taking the summatory of the functions $I_{i}$ our conclusion follows. 
Lemma 0.6 With the same hypotheses as Lemma 0.3 we have

$$
\begin{aligned}
\frac{d}{d t} \mathcal{N}_{1}(t) \leq & -\frac{K(0) L_{0}}{4}\left\{a\left|U_{x}\left(L_{0}, t\right)\right|^{2}+\left|U_{t}\left(L_{0}, t\right)\right|^{2}+\left|\Theta\left(L_{0}, t\right)\right|^{2}\right\} \\
& -\frac{L_{0}}{4}\left|K * \Theta_{x}(0, t)\right|^{2}+\frac{K(0)}{2} \int_{0}^{L_{0}}\left|U_{t}\right|^{2} d x+a K(0) \int_{0}^{L_{0}}\left|U_{x}\right|^{2} d x \\
& +K(0) \int_{0}^{L_{0}}|\Theta|^{2} d x+\frac{1}{2} \int_{0}^{L_{0}}\left|K * \Theta_{x}\right|^{2} d x \\
& +\left(\frac{L_{0}^{2}}{8 K(0)}+\frac{\alpha^{2} L_{0}^{2}}{8 a K(0)}\right) \int_{0}^{L_{0}}\left|K^{\prime} * \Theta_{x}\right|^{2} d x \\
& +c \gamma\left\{E_{1}(t ; U, \Theta)+\int_{0}^{L_{0}}\left|K * \Theta_{x}\right|^{2} d x\right\}
\end{aligned}
$$

where $\mathcal{N}_{1}(t)=\int_{0}^{L_{0}}\left(\frac{L_{0}}{2}-x\right)\left\{K(0) U_{t} U_{x}+\Theta K * \Theta_{x}+\alpha U_{x} K * \Theta_{x}\right\} d x$

Proof: Multiplying (15) by $\varphi(x) U_{x}$ and integrating from 0 to $L_{0}$ we get

$$
\begin{aligned}
\frac{d}{d t} \int_{0}^{L_{0}} \varphi(x) U_{t} U_{x} d x= & \frac{1}{2} \int_{0}^{L_{0}} \varphi(x) \frac{d}{d x}\left|U_{t}\right|^{2} d x+\frac{a}{2} \int_{0}^{L_{0}} \varphi(x) \frac{d}{d x}\left|U_{x}\right|^{2} d x \\
& -\alpha \int_{0}^{L_{0}} \Theta_{x} \varphi(x) U_{x} d x+\int_{0}^{L_{0}} R \varphi(x) U_{x} d x \\
= & \frac{\varphi\left(L_{0}\right)}{2}\left\{a\left|U_{x}\left(L_{0}, t\right)\right|^{2}+\left|U_{t}\left(L_{0}, t\right)\right|^{2}\right\} \\
& -\frac{a \varphi(0)}{2}\left|U_{x}(0, t)\right|^{2}-\frac{1}{2} \int_{0}^{L_{0}} \varphi^{\prime}(x)\left\{\left|U_{t}\right|^{2}+a\left|U_{x}\right|^{2}\right\} d x \\
& -\alpha \int_{0}^{L_{0}} \Theta_{x} \varphi(x) U_{x} d x+\int_{0}^{L_{0}} R \varphi(x) U_{x} d x .
\end{aligned}
$$

Now, observe that

$$
\begin{aligned}
\frac{d}{d t} \int_{0}^{L_{0}} \varphi(x) K * \Theta_{x} \Theta d x= & \frac{K(0)}{2} \int_{0}^{L_{0}} \varphi(x) \frac{d}{d x}|\Theta|^{2} d x+\int_{0}^{L_{0}} \varphi(x) K^{\prime} * \Theta_{x} \Theta d x \\
& +\frac{1}{2} \int_{0}^{L_{0}} \varphi(x) \frac{d}{d x}\left|K * \Theta_{x}\right|^{2} d x-\alpha \int_{0}^{L_{0}} \varphi(x) K * \Theta_{x} U_{x t} d x \\
& +\int_{0}^{L_{0}} S \varphi(x) K * \Theta_{x} d x . \\
= & \frac{K(0) \varphi\left(L_{0}\right)}{2}\left|\Theta\left(L_{0}, t\right)\right|^{2}-\frac{K(0)}{2} \int_{0}^{L_{0}} \varphi^{\prime}(x)|\Theta|^{2} d x \\
& +\int_{0}^{L_{0}} \varphi(x) K^{\prime} * \Theta_{x} \Theta d x-\frac{\varphi(0)}{2}\left|K * \Theta_{x}(0, t)\right|^{2} \\
& -\frac{1}{2} \int_{0}^{L_{0}} \varphi^{\prime}(x)\left|K * \Theta_{x}\right|^{2} d x-\alpha \frac{d}{d t} \int_{0}^{L_{0}} \varphi(x) K * \Theta_{x} U_{x} d x \\
& +\alpha \int_{0}^{L_{0}} \varphi(x) K(0) \Theta_{x} U_{x} d x+\alpha \int_{0}^{L_{0}} \varphi(x) K^{\prime} * \Theta_{x} U_{x} d x \\
& +\int_{0}^{L_{0}} S \varphi(x) K * \Theta_{x} d x .
\end{aligned}
$$


Therefore

$$
\begin{aligned}
& \frac{d}{d t} \int_{0}^{L_{0}} \varphi(x) K * \Theta_{x}\left[\Theta+\alpha U_{x}\right] d x \\
= & \frac{K(0) \varphi\left(L_{0}\right)}{2}\left|\Theta\left(L_{0}, t\right)\right|^{2}-\frac{K(0)}{2} \int_{0}^{L_{0}} \varphi^{\prime}(x)|\Theta|^{2} d x \\
& +\int_{0}^{L_{0}} \varphi(x) K^{\prime} * \Theta_{x} \Theta d x-\frac{\varphi(0)}{2}\left|K * \Theta_{x}(0, t)\right|^{2} \\
& -\frac{1}{2} \int_{0}^{L_{0}} \varphi^{\prime}(x)\left|K * \Theta_{x}\right|^{2} d x+\alpha K(0) \int_{0}^{L_{0}} \varphi(x) \Theta_{x} U_{x} d x \\
& +\alpha \int_{0}^{L_{0}} \varphi(x) K^{\prime} * \Theta_{x} U_{x} d x+\int_{0}^{L_{0}} S \varphi(x) K * \Theta_{x} d x .
\end{aligned}
$$

Taking $\varphi(x)=\left(\frac{L_{0}}{2}-x\right)$ in above identies and using the estimate

$$
\begin{gathered}
\left|\alpha \int_{0}^{L_{0}}\left(\frac{L_{0}}{2}-x\right) U_{x} K^{\prime} * \Theta_{x} d x\right| \leq \frac{K(0) a}{2} \int_{0}^{L_{0}}\left|U_{x}\right|^{2} d x+\frac{L_{0}^{2} \alpha^{2}}{8 a K(0)} \int_{0}^{L_{0}}\left|K^{\prime} * \Theta_{x}\right|^{2} d x \\
\left|\int_{0}^{L_{0}}\left(\frac{L_{0}}{2}-x\right) \Theta K^{\prime} * \Theta_{x} d x\right| \leq \frac{K(0)}{2} \int_{0}^{L_{0}}|\Theta|^{2} d x+\frac{L_{0}^{2}}{8 K(0)} \int_{0}^{L_{0}}\left|K^{\prime} * \Theta_{x}\right|^{2} d x .
\end{gathered}
$$

our conclusion follows.

To show the uniform rate decay we will use a result due to Kim [6]

Lemma 0.7 Let us denote $\left\{z^{k}\right\}$ a sequence of function satisfying

$$
\begin{array}{lll}
z^{k} \stackrel{*}{\rightarrow} z & \text { in } & L^{\infty}\left(0, T ; H^{p}\left(0, L_{0}\right)\right) \\
z_{t}^{k} \stackrel{*}{\rightarrow} z_{t} & \text { in } & L^{\infty}\left(0, T ; H^{q}\left(0, L_{0}\right)\right)
\end{array}
$$

when $k \rightarrow \infty$, for $p<q$. Then we have for any $r<q$ that

$$
z^{k} \rightarrow z \quad \text { in } \quad C\left(0, T ; H^{r}\left(0, L_{0}\right)\right)
$$

Proof. See [6].

Lemma 0.8 With the same hypotheses as Lemma 0.3 we have, for any $\delta>0$ exist $C_{\delta}>0$ indepedent of initial data such that to $T$ large enough

$$
\int_{0}^{T}\left[\left|U\left(L_{0}, t\right)\right|^{2}+\left\|q_{x}(., t)\right\|_{H^{\frac{3}{4}}}^{2}\right] d t \leq \delta \int_{0}^{T} E(t ; U, \Theta, V) d t+C_{\delta} \int_{0}^{T} \int_{0}^{L}\left|K * \Theta_{x}\right|^{2} d x d t
$$

for any solution $(U, \Theta, V)$ of system $(15)-(17)$ 
Proof: We reason by contradiction. Let us suppose that there exists a sequence of initial data $\left(U_{0}^{\nu}, \Theta_{0}^{\nu}, V_{0}^{\nu}\right),\left(U_{1}^{\nu}, V_{1}^{\nu}\right)$ and positive constant $\delta_{0}>0$ such that the corresponding solution of system

$$
\begin{aligned}
U_{t t}^{\nu}-a U_{x x}^{\nu}+\alpha \Theta_{x}^{\nu} & =2 \gamma U_{t}^{\nu}-\gamma^{2} U^{\nu} \\
\Theta_{t}^{\nu}-K * \Theta_{x x}^{\nu}+\alpha U_{x t}^{\nu} & =\gamma \Theta^{\nu}+\alpha \gamma U_{x}^{\nu}, \\
V_{t t}^{\nu}-\beta V_{x x}^{\nu} & =2 \gamma V_{t}^{\nu}-\gamma^{2} V^{\nu} \\
U^{\nu}(x, 0)=U_{0}^{\nu}(x), & U_{t}^{\nu}(x, 0)=U_{1}^{\nu}(x), \quad \Theta^{\nu}(x, 0)=\Theta_{0}^{\nu}(x), \\
V^{\nu}(x, 0)=V_{0}^{\nu}(x), \quad V_{t}^{\nu}(x, 0) & =V_{1}^{\nu}(x),
\end{aligned}
$$

with the following boundary condition

$$
U^{\nu}(0, t)=\Theta^{\nu}(0, t)=V^{\nu}(L, t)=\Theta_{x}^{\nu}\left(L_{0}, t\right)=0 \quad \forall t>0
$$

and transmission's condition

$$
\begin{aligned}
\beta V_{x}^{\nu}\left(L_{0}, t\right) & =a U_{x}^{\nu}\left(L_{0}, t\right)-\alpha \Theta^{\nu}\left(L_{0}, t\right) \quad \forall t>0 \\
U^{\nu}\left(L_{0}, t\right) & =V^{\nu}\left(L_{0}, t\right) \quad \forall t>0
\end{aligned}
$$

satisfying

$$
\int_{0}^{T}\left[\left|U^{\nu}\left(L_{0}, t\right)\right|^{2}+\left\|q_{x}^{\nu}(., \tau)\right\|_{H^{\frac{3}{4}}}^{2}\right] d t=1
$$

where $q^{\nu}$ is solution of

$$
\begin{aligned}
-q_{x x}^{\nu} & =U_{t}^{\nu} & & \text { in }\left(0, L_{0}\right) \times \mathbb{R}^{+} \\
q^{\nu}(0, t) & =q^{\nu}\left(L_{0}, t\right)=0 & & \text { in } \mathbb{R}^{+}
\end{aligned}
$$

and verifying the following inequality

$$
1 \geq \delta_{0} \int_{0}^{T} E\left(t ; U^{\nu}, \Theta^{\nu}, V^{\nu}\right) d t+\nu \int_{0}^{T} \int_{0}^{L}\left|K * \Theta_{x}^{\nu}\right|^{2} d x d t
$$

It follows that

$$
\int_{0}^{T} E\left(t ; U^{\nu}, \Theta^{\nu}, V^{\nu}\right) d t \text { is bounded for any } \nu \in \mathbb{N}
$$

and also that

$$
K * \Theta_{x}^{\nu} \rightarrow 0 \quad \text { in } \quad L^{2}\left(0, T ; L^{2}(0, L)\right)
$$


From (29) we have there exist a subsequence of $\left(U^{\nu}, \Theta^{\nu}, V^{\nu}\right)_{\nu \in \mathbb{N}}$, which we denote in the same way, such that

$$
\left\{\begin{array}{ccc}
U_{t}^{\nu} \stackrel{*}{*} U_{t} & \text { in } & L^{\infty}\left(0, T ; L^{2}\left(0, L_{0}\right)\right) \\
\Theta^{\nu} \stackrel{*}{*} \Theta & \text { in } & L^{\infty}\left(0, T ; L^{2}\left(0, L_{0}\right)\right) \\
V_{t}^{\nu} \stackrel{*}{\rightarrow} V_{t} & \text { in } & L^{\infty}\left(0, T ; L^{2}\left(L_{0}, L\right)\right) \\
U_{x}^{\nu} \stackrel{*}{*} U_{x} & \text { in } & L^{\infty}\left(0, T ; L^{2}\left(0, L_{0}\right)\right) \\
V_{x}^{\nu} \stackrel{*}{\rightarrow} V_{x} & \text { in } & L^{\infty}\left(0, T ; L^{2}\left(L_{0}, L\right)\right)
\end{array}\right.
$$

Since $K * \Theta^{\nu}(0, t)=0$ for any $\nu$ and any $t$ we get that $K * \Theta(0, t)=0$ and (30) implies $K * \Theta_{x}=0$. So we get that $K * \Theta=0$. Therefore, using the Volterra's resolvent we conclude $\Theta=0$. From $-q_{x x}^{\nu}=U_{t}^{\nu}$ and (31) it follows that

$$
\left(q_{x}^{\nu}, q_{t}^{\nu}\right) \quad \text { is bounded in } \quad\left[L^{2}\left(0, T ; H^{1}\left(0, L_{0}\right)\right)\right]^{2} .
$$

Applying Lions-Aubin's Theorem (See [7] ) we conclude that

$$
q_{x}^{\nu} \rightarrow q_{x} \quad \text { in } \quad L^{2}\left(0, T ; H^{\frac{3}{4}}\left(0, L_{0}\right)\right) .
$$

On the other side, from (31) and Lemma 0.7 it follows that

$$
U^{\nu} \rightarrow U \quad \text { in } \quad C\left(0, T ; H^{r}\left(0, L_{0}\right)\right)
$$

for $r<1$. Using (32) and (33) in (28) we have

$$
\int_{0}^{T}\left[\left|U\left(L_{0}, t\right)\right|^{2}+\left\|q_{x}(., t)\right\|_{H^{\frac{3}{4}}}^{2}\right] d t=1 .
$$

Substituing $\Theta=0$ in (26) we have

$$
U_{x t}^{\nu}=\gamma U_{x}^{\nu}
$$

Since $U(0, t)=0$ we conclude that

$$
U(x, t)=U(x, 0) e^{\gamma t}
$$

Using this fact in (25) we have that

$$
-a U_{x x}=0
$$

Since $U(0, t)=0$ then we can write $U(x, t)=c x e^{\gamma t}$. On the other hand $V$ satisfies

$$
\begin{gathered}
V_{t t}-\beta V_{x x}=2 \gamma V_{t}-\gamma^{2} V \\
V\left(L_{0}, t\right)=c L_{0} e^{\gamma t}, \quad \beta V_{x}\left(L_{0}, t\right)=a c e^{\gamma t}, \quad V(L, t)=0
\end{gathered}
$$

Let us denote by $v(x, t)=e^{-\gamma t} V$ we have

$$
v_{t t}-\beta v_{x x}=0
$$




$$
v\left(L_{0}, t\right)=c L_{0}, \quad \beta v_{x}\left(L_{0}, t\right)=a c, \quad v(L, t)=0 .
$$

Differentiating with respect to $t$ and denoting by $W=v_{t}$ we have that

$$
\begin{gathered}
W_{t t}-\beta W_{x x}=0 \\
W\left(L_{0}, t\right)=0, \quad W_{x}\left(L_{0}, t\right)=0, \quad W(L, t)=0 .
\end{gathered}
$$

Therefore we can extend the solution in the following way

$$
\bar{W}=\left\{\begin{array}{ll}
0 & x \in\left[0, L_{0}\right], \quad t>0 \\
W & x \in\left[L_{0}, L\right], \quad t>0
\end{array} .\right.
$$

Since $\bar{W} \in H^{1}(0, L)$ then $\bar{W}$ is a weak solution of the wave equation vanishing over $\left[0, L_{0}\right]$. Therefore from Holgrem's Theorem (See [5]) we conclude that $\bar{W}=0$, which implies that $v_{t}=0$, therefore $V(x, t)=\varphi(x) e^{\gamma t}$. Then we get the following Elliptic transmission problem

$$
\begin{gathered}
-a U_{x x}=0 \\
-\beta V_{x x}=0 \\
U\left(L_{0}, t\right)=V\left(L_{0}, t\right), \quad a U_{x}\left(L_{0}, t\right)=\beta V_{x}\left(L_{0}, t\right) \quad U(0, t)=0, \quad V(L, t)=0 .
\end{gathered}
$$

Multiplying equation (35) by $U$ and equation (36) by $V$ we get

$$
\begin{gathered}
a \int_{0}^{L_{0}}\left|U_{x}\right|^{2} d x-\left.a U_{x} U\right|_{0} ^{L_{0}}=0 \\
\beta \int_{L_{0}}^{L}\left|V_{x}\right|^{2} d x-\left.\beta V_{x} V\right|_{L_{0}} ^{L}=0
\end{gathered}
$$

Using the transmission conditions, we conclude that

$$
U=V=0
$$

Therefore the convergence in (32) and (33) implies that

$$
q_{x}=U=0 \quad \text { a.e. } \quad \text { in } \quad(0, T) \times\left(0, L_{0}\right) .
$$

Hence, we arrive to

$$
\int_{0}^{T}\left[\left|U\left(L_{0}, t\right)\right|^{2}+\left\|q_{x}(., \tau)\right\|_{H^{\frac{3}{4}}}^{2}\right] d t=0
$$

But this is contradictory with (34). The proof is now complete.

The exponential decay is summarized in the following Theorem. 
Theorem 0.2 Let us suppose that the initial data satisfy

$$
\left(u_{0}, v_{0}\right) \in\left[H^{2}\left(0, L_{0}\right) \times H^{2}\left(L_{0}, L\right)\right] \cap \mathcal{V}, \quad\left(u_{1}, v_{1}\right) \in \mathcal{V}, \quad \theta_{0} \in H^{1}\left(0, L_{0}\right)
$$

verifying the compatibility condition given by

$$
a u_{0, x}\left(L_{0}\right)-\alpha \theta_{0}\left(L_{0}\right)=\beta v_{0, x}\left(L_{0}\right)
$$

there exist positive constant $C_{0}$ and $\gamma$ such that

$$
E(t ; u, \theta, v) \leq C_{0} E(0 ; u, \theta, v) e^{-\gamma t} .
$$

Proof: From Lemmas 0.5 and 0.6 we have

$$
\begin{aligned}
\frac{d}{d t}\left[\int_{0}^{L_{0}} q_{x} \Theta d x+\frac{\alpha}{8 K(0)} \mathcal{N}_{1}(t)\right] \leq & -\frac{7 \alpha L_{0}}{256}\left\{a\left|U_{x}\left(L_{0}, t\right)\right|^{2}+\left|U_{t}\left(L_{0}, t\right)\right|^{2}+\left|\Theta\left(L_{0}, t\right)\right|^{2}\right\} \\
& -\frac{7 \alpha}{16} \int_{0}^{L_{0}}\left|U_{t}\right|^{2} d x+\frac{5 \alpha a}{32} \int_{0}^{L_{0}}\left|U_{x}\right|^{2} d x \\
& +\left(\frac{9 \alpha}{8}+\frac{16 a c_{p}^{2} \delta_{1}}{\alpha L_{0}}+\frac{16 a}{\alpha}\right) \int_{0}^{L_{0}}|\Theta|^{2} d x \\
& +\left(\frac{1}{2 \alpha}+\frac{\alpha}{16 K(0)}\right) \int_{0}^{L_{0}}\left|K * \Theta_{x}\right|^{2} d x \\
& +c_{0} \int_{0}^{L_{0}}\left|K^{\prime} * \Theta_{x}\right|^{2} d x \\
& +\left(\frac{64 \alpha \lambda_{1}^{2}}{L_{0}}+\frac{16 \lambda_{1}^{2} K(0)}{\alpha L_{0}}\right)\left\|q_{x}(., t)\right\|_{H^{3 / 4}}^{2} \\
& +c \gamma\left\{E_{1}(t ; U, \Theta)+\int_{0}^{L_{0}}\left|K * \Theta_{x}\right|^{2} d x\right\}
\end{aligned}
$$

where $c_{0}=\left(\frac{L_{0}^{2} \alpha}{64 K(0)^{2}}+\frac{\alpha^{3} L_{0}^{2}}{64 a K(0)^{2}}\right)$.

Let us introduce the functional

$$
\mathcal{N}_{2}(t)=\int_{0}^{L_{0}} q_{x} \Theta d x+\frac{\alpha}{8 K(0)} \mathcal{N}_{1}(t)+\frac{3 \alpha}{8} \int_{0}^{L_{0}} U U_{t} d x .
$$

From last inequality and (21) of the Lemma 0.4 we get

$$
\begin{aligned}
\frac{d}{d t} \mathcal{N}_{2}(t) \leq & -\frac{3 \alpha L_{0}}{128}\left\{a\left|U_{x}\left(L_{0}, t\right)\right|^{2}+\left|U_{t}\left(L_{0}, t\right)\right|^{2}+\left|\Theta\left(L_{0}, t\right)\right|^{2}\right\} \\
& -\frac{\alpha a}{32} \int_{0}^{L_{0}}\left|U_{x}\right|^{2} d x-\frac{\alpha}{16} \int_{0}^{L_{0}}\left|U_{t}\right|^{2} d x+c_{0} \int_{0}^{L_{0}}\left|K^{\prime} * \Theta_{x}\right|^{2} d x \\
& +c_{1}\left|U\left(L_{0}, t\right)\right|^{2}+\left(\frac{64 \alpha \lambda_{1}^{2}}{L_{0}}+\frac{16 \lambda_{1}^{2} K(0)}{\alpha L_{0}}\right)\left\|q_{x}(., t)\right\|_{H^{3 / 4}}^{2} \\
& +c_{2} \int_{0}^{L_{0}}|\Theta|^{2} d x+c_{3} \int_{0}^{L_{0}}\left|K * \Theta_{x}\right|^{2} d x \\
& +c \gamma\left\{E_{1}(t ; U, \Theta)+\int_{0}^{L_{0}}\left|K * \Theta_{x}\right|^{2} d x\right\}
\end{aligned}
$$


where

$$
\begin{gathered}
c_{1}=\frac{3 \alpha}{8}\left(\frac{24 a}{L_{0}}+\frac{24 \alpha^{2}}{L_{0}}\right), \quad c_{2}=\left(\frac{9 \alpha}{8}+\frac{16 a c_{p}^{2} \delta_{1}}{\alpha L_{0}}+\frac{16 a}{\alpha}+\frac{3 \alpha^{3}}{16 a}\right), \\
c_{3}=\left(\frac{1}{2 \alpha}+\frac{\alpha}{16 K(0)}\right) .
\end{gathered}
$$

Note that

$$
\begin{gathered}
\left|K * \Theta\left(L_{0}, t\right)\right|^{2} \leq c_{p}^{2} \int_{0}^{L_{0}}\left|K * \Theta_{x}\right|^{2} d x \\
\left|\alpha \int_{0}^{L_{0}} U_{t} K * \Theta_{x} d x\right| \leq \frac{\alpha K(0)}{96 c_{2}} \int_{0}^{L_{0}}\left|U_{t}\right|^{2} d x+\frac{24 c_{2}}{K(0)} \int_{0}^{L_{0}}\left|K * \Theta_{x}\right|^{2} d x, \\
\left|\alpha U_{t}\left(L_{0}, t\right) K * \Theta\left(L_{0}, t\right)\right| \leq \frac{\alpha L_{0} K(0)}{768 c_{2}}\left|U_{t}\left(L_{0}, t\right)\right|^{2}+\frac{192 \alpha c_{2} c_{p}^{2}}{L_{0} K(0)} \int_{0}^{L_{0}}\left|K * \Theta_{x}\right|^{2} d x, \\
\left|\int_{0}^{L_{0}} \Theta K^{\prime} * \Theta d x\right| \leq \frac{K(0)}{2} \int_{0}^{L_{0}}|\Theta|^{2} d x+\frac{c_{p}^{2}}{2 K(0)} \int_{0}^{L_{0}}\left|K^{\prime} * \Theta_{x}\right|^{2} d x .
\end{gathered}
$$

Using the above estimate in (20) from Lemma 0.4 it follows

$$
\begin{aligned}
-\frac{d}{d t} \int_{0}^{L_{0}} K * \Theta \Theta d x \leq & -\frac{K(0)}{2} \int_{0}^{L_{0}}|\Theta|^{2} d x+\frac{\alpha L_{0} K(0)}{768 c_{2}}\left|U_{t}\left(L_{0}, t\right)\right|^{2} \\
& +\frac{\alpha K(0)}{96 c_{2}} \int_{0}^{L_{0}}\left|U_{t}\right|^{2} d x+\frac{c_{p}^{2}}{2 K(0)} \int_{0}^{L_{0}}\left|K^{\prime} * \Theta_{x}\right|^{2} d x \\
& +\left(1+\frac{192 \alpha c_{2} c_{p}^{2}}{L_{0} K(0)}+\frac{24 c_{2}}{K(0)}\right) \int_{0}^{L_{0}}\left|K * \Theta_{x}\right|^{2} d x \\
& +c \gamma\left\{E_{1}(t ; U, \Theta)+\int_{0}^{L_{0}}\left|K * \Theta_{x}\right|^{2} d x\right\}
\end{aligned}
$$

Let us write

$$
\mathcal{N}_{3}(t)=\mathcal{N}_{2}(t)-\frac{3 c_{2}}{K(0)} \int_{0}^{L_{0}} K * \Theta \Theta d x .
$$

From (37) and (38) we get

$$
\begin{aligned}
\frac{d}{d t} \mathcal{N}_{3}(t) \leq & -\frac{5 \alpha L_{0}}{256}\left\{a\left|U_{x}\left(L_{0}, t\right)\right|^{2}+\left|U_{t}\left(L_{0}, t\right)\right|^{2}+\left|\Theta\left(L_{0}, t\right)\right|^{2}\right\} \\
& -\frac{\alpha a}{32} \int_{0}^{L_{0}}\left|U_{x}\right|^{2} d x-\frac{\alpha}{32} \int_{0}^{L_{0}}\left|U_{t}\right|^{2} d x-\frac{c_{2}}{2} \int_{0}^{L_{0}}|\Theta|^{2} d x \\
& +c_{1}\left|U\left(L_{0}, t\right)\right|^{2}+c_{4} \int_{0}^{L_{0}}\left|K * \Theta_{x}\right|^{2} d x+c_{5} \int_{0}^{L_{0}}\left|K^{\prime} * \Theta_{x}\right|^{2} d x \\
& +c_{6}\left\|q_{x}(., t)\right\|_{H^{3 / 4}}^{2}+c \gamma\left\{E_{1}(t ; U, \Theta)+\int_{0}^{L_{0}}\left|K * \Theta_{x}\right|^{2} d x\right\}
\end{aligned}
$$

where

$$
c_{4}=\left[c_{3}+\frac{3 c_{2}}{K(0)}\left(1+\frac{192 \alpha c_{2} c_{p}^{2}}{L_{0} K(0)}+\frac{24 c_{2}}{K(0)}\right)\right], \quad c_{5}=\left(c_{0}+\frac{3 c_{2} c_{p}^{2}}{2 K(0)^{2}}\right)
$$




$$
c_{6}=\left(\frac{64 \alpha \lambda_{1}^{2}}{L_{0}}+\frac{16 \lambda_{1}^{2} K(0)}{\alpha L_{0}}\right) .
$$

Let us introduce the following functional:

$$
\mathcal{N}_{4}(t)=\mathcal{N}_{3}(t)+\frac{\alpha L_{0}}{64 \lambda_{0}} \int_{L_{0}}^{L}(x-L) V_{x} V_{t} d x .
$$

From (22) of the Lemma 0.4 and last inequality

$$
\begin{aligned}
\frac{d}{d t} \mathcal{N}_{4}(t) \leq & -\mu_{0} E(t ; U, \Theta, V) \\
& +\mu_{1}\left(\left|U\left(L_{0}, t\right)\right|^{2}+\left\|q_{x}(., t)\right\|_{H^{3 / 4}}^{2}\right) \\
& +c_{4} \int_{0}^{L_{0}}\left|K * \Theta_{x}\right|^{2} d x+c_{5} \int_{0}^{L_{0}}\left|K^{\prime} * \Theta_{x}\right|^{2} d x \\
& +c \gamma\left\{E(t ; U, \Theta, V)+\int_{0}^{L_{0}}\left|K * \Theta_{x}\right|^{2} d x\right\}
\end{aligned}
$$

where $\mu_{0}=C\left(\alpha, a, L_{0}, \beta, \lambda_{0}, c_{p}, \delta_{1}\right)$ and $\mu_{1}=\max \left\{c_{1}+\frac{\alpha L_{0} \bar{c}}{256 \lambda_{0}^{2}}, c_{6}\right\}$. Now we define

$$
\begin{aligned}
\mathcal{L}(t) & =\mathcal{N}_{4}(t)+N E(t ; U, \Theta, V) \\
\mathcal{F}(t) & =\mathcal{L}(t)+\int_{0}^{L_{0}}\left|K * \Theta_{x}\right|^{2} d x
\end{aligned}
$$

Observe that

$$
\begin{array}{r}
\frac{d}{d t} \int_{0}^{L_{0}}\left|K * \Theta_{x}\right|^{2} d x=2 \int_{0}^{L_{0}} K^{\prime} * \Theta_{x} K * \Theta_{x} d x+2 K(0) \int_{0}^{L_{0}} K * \Theta_{x} \Theta_{x} d x \\
\quad \leq \int_{0}^{L_{0}}\left|K^{\prime} * \Theta_{x}\right|^{2} d x+\int_{0}^{L_{0}}\left|K * \Theta_{x}\right|^{2} d x+2 K(0) \int_{0}^{L_{0}} K * \Theta_{x} \Theta_{x} d x
\end{array}
$$

From this, Lemma 0.3 and (40) we get

$$
\begin{aligned}
\frac{d}{d t} \mathcal{F}(t) \leq & -\mu_{0} E(t ; U, \Theta, V)+\mu_{1}\left(\left|U\left(L_{0}, t\right)\right|^{2}+\left\|q_{x}(., t)\right\|_{H^{3 / 4}}^{2}\right) \\
& +\left(c_{4}+1\right) \int_{0}^{L_{0}}\left|K * \Theta_{x}\right|^{2} d x+\left(c_{5}+1\right) \int_{0}^{L_{0}}\left|K^{\prime} * \Theta_{x}\right|^{2} d x \\
& -(N-2 K(0)) \int_{0}^{L_{0}} K * \Theta_{x} \Theta_{x} d x+N c \gamma E(t ; U, \Theta, V) \\
& +c \gamma\left\{E(t ; U, \Theta, V)+\int_{0}^{L_{0}}\left|K * \Theta_{x}\right|^{2} d x\right\} .
\end{aligned}
$$

Integrating from 0 to $t$ the above relation, using Lemma 0.8 we have

$$
\begin{aligned}
\mathcal{F}(t)-\mathcal{F}(0) \leq & -\mu_{0} \int_{0}^{t} E(\tau ; U, \Theta, V) d \tau+\mu_{1} \delta \int_{0}^{t} E(\tau ; U, \Theta, V) d \tau \\
& -(N-2 K(0)) \int_{0}^{t} \int_{0}^{L_{0}} K * \Theta_{x} \Theta_{x} d x d \tau
\end{aligned}
$$




$$
\begin{aligned}
& +\left(\mu_{1} C_{\delta}+c_{4}+1\right) \int_{0}^{t} \int_{0}^{L_{0}}\left|K * \Theta_{x}\right|^{2} d x d \tau \\
& +\left(c_{5}+1\right) \int_{0}^{t} \int_{0}^{L_{0}}\left|K^{\prime} * \Theta_{x}\right|^{2} d x d \tau+N c \gamma \int_{0}^{t} E(\tau ; U, \Theta, V) d \tau \\
& +c \gamma \int_{0}^{t}\left[E(\tau ; U, \Theta, V)+\int_{0}^{L_{0}}\left|K * \Theta_{x}\right|^{2} d x\right] d \tau .
\end{aligned}
$$

Observe that, using Lemma 0.1 , hypotheses on $k$ and $k^{\prime}$ we get

$$
\begin{aligned}
& \int_{0}^{t} \int_{0}^{L_{0}} K * \Theta_{x} \Theta_{x} d x d \tau \geq C \int_{0}^{t} \int_{0}^{L_{0}}\left|K * \Theta_{x}\right|^{2} d x d \tau \\
& \int_{0}^{t} \int_{0}^{L_{0}}\left|K^{\prime} * \Theta_{x}\right|^{2} d x d \tau \leq C \int_{0}^{t} \int_{0}^{L_{0}}\left|K * \Theta_{x}\right|^{2} d x d \tau
\end{aligned}
$$

From this and choosing $N>4 K(0)$ we conclude that

$$
\begin{aligned}
\mathcal{F}(t)-\mathcal{F}(0) \leq & -\mu_{0} \int_{0}^{t} E(\tau ; U, \Theta, V) d \tau+\mu_{1} \delta \int_{0}^{t} E(\tau ; U, \Theta, V) d \tau \\
& -\left(\frac{N C}{2}-\mu_{1} C_{\delta}-c_{4}-1-c_{5} C-C+c \gamma\right) \int_{0}^{t} \int_{0}^{L_{0}}\left|K * \Theta_{x}\right|^{2} d x d \tau \\
& +(N+1) c \gamma \int_{0}^{t} E(\tau ; U, \Theta, V) d \tau
\end{aligned}
$$

Choose $\delta>0$ such that $\delta \mu_{1}<\frac{\mu_{0}}{4}$. Taking $N$ large enough and $\gamma$ sufficiently small we conclude that

$$
\mathcal{F}(t)-\mathcal{F}(0) \leq-\frac{\mu_{0}}{2} \int_{0}^{t}\left[E(\tau ; U, \Theta, V)+\int_{0}^{L_{0}}\left|K * \Theta_{x}\right|^{2} d x\right] d \tau<0
$$

and

$$
\begin{aligned}
& \mathcal{L}(t) \leq k_{1}\left[E(t ; U, \Theta, V)+\int_{0}^{L_{0}}\left|K * \Theta_{x}\right|^{2} d x\right] \\
& \mathcal{L}(t) \geq k_{2}\left[E(t ; U, \Theta, V)-\int_{0}^{L_{0}}\left|K * \Theta_{x}\right|^{2} d x\right]
\end{aligned}
$$

where $k_{1}$ and $k_{2}$ are positive constants.

Since

$$
\begin{aligned}
E(t ; U, \Theta, V) & \leq \frac{1}{k_{2}}\left[\mathcal{L}(t)+\int_{0}^{L_{0}}\left|K * \Theta_{x}\right|^{2} d x\right] \\
& \leq \frac{1}{k_{2}} \mathcal{F}(t) \leq \frac{1}{k_{2}} \mathcal{F}(0) \\
& \leq \frac{1}{k_{2}} \mathcal{L}(0) \leq \frac{k_{1}}{k_{2}} E(0 ; U, \Theta, V) .
\end{aligned}
$$

Our conclusion follows. The proof is now complete. 


\section{References}

[1] B.D. Coleman, M.E. Gurtin, Equipresence and constitutive equations for rigid heat conductors, Z. Angew. Math. Phys 18, 199-208 (1967).

[2] M. Fabrizio and B. Lazzari, On the existence and asymptotic stability of solutions for linearly viscoelastic solids, Arch. Rat. Mech. Anal. 116, 139-152 (1991).

[3] C. Giorgi, M. G. Naso; On the exponential stability of linear non-Fourier thermoviscoelastic bar, quaderni del Seminario Matematico di Brescia 2/97, (1997).

[4] M.E. Gurtin, A.C. Pipkin, A general theory of heat conduction with finite wave speeds, Arch. Rational Mech. Anal. 31, 113-126 (1968).

[5] L.Hörmander, Linear partial differential operators, Springer Verlag, New York, (1976).

[6] J.U.Kim, A boundary thin obstacle problem for a wave equation. Commun. in Partial Differential Equations, 14(8\&9), 1011-1026 (1989).

[7] , J. L. Lions, Quelques methodes de résolution des problénes aux limites non linéaires. Dunod, Paris 1969.

[8] J.L. Lions, Contrôlabilité exacte perturbations et stabilisation de systèmes distribués. Collection RMA, Masson Paris 1988 (tome 1).

[9] J.E. Muñoz Rivera; Asymptotic Behaviour in Linear Viscoelasticity. Quarterly of Applied Mathematics, III-4, 629-648 (1994).

[10] O. J. Staffans; On a nonlinear hyperbolic Volterra Equation. Siam J. Math, Anal. 11, 5, 793-812 (1980). 